\title{
New Consequences of Induced Transparency in a Double- $\Lambda$ scheme: Destructive Interference In Four-wave Mixing
}

\author{
M.G. Payne \\ Department of Physics, Georgia Southern University, Statesboro, GA \\ Lu Deng \\ Electron and Optical Physics Division, NIST, Gaithersburg, MD
}

(Dated: October 29, 2018)

\begin{abstract}
We investigate a four-state system interacting with long and short laser pulses in a weak probe beam approximation. We show that when all lasers are tuned to the exact unperturbed resonances, part of the four-wave mixing (FWM) field is strongly absorbed. The part which is not absorbed has the exact intensity required to destructively interfere with the excitation pathway involved in producing the FWM state. We show that with this three-photon destructive interference, the conversion efficiency can still be as high as $25 \%$. Contrary to common belief, our calculation shows that this process, where an ideal one-photon electromagnetically induced transparency is established, is not most suitable for high efficiency conversion. With appropriate phase-matching and propagation distance, and when the three-photon destructive interference does not occur, we show that the photon flux conversion efficiency is independent of probe intensity and can be close to $100 \%$. In addition, we show clearly that the conversion efficiency is not determined by the maximum atomic coherence between two lower excited states, as commonly believed. It is the combination of phase-matching and constructive interference involving the two terms arising in producing the mixing wave that is the key element for the optimized FWM generation. Indeed, in this scheme no appreciable excited state is produced, so that the atomic coherence between states $\mid 0>$ and $\mid 2>$ is always very small.
\end{abstract}

\section{INTRODUCTION}

Efficient four-wave mixing (FWM) processes in the context of electromagnetically induced transparency (EIT) [1] involving a double- $\Lambda$ scheme have been the subject of several recent studies [2-4]. In almost all of these studies, a large atomic coherence has been considered to be a key player in yielding a high conversion efficiency. Therefore, maximum atomic coherence was assumed. Furthermore, all studies available so far are based on either a steady-state approximation[2-4] or a full-numerical calculation[3] to obtain predictions on the system. A numerical calculation usually fails to yield as much insight into a problem as would be obtained from an analytical solution. The steadystate treatment is analytical, but it oversimplifies the problem and, in some cases, it leads to incorrect conclusions. In particular, it is known that a steady-state treatment of a four-level double- $\Lambda$ scheme can lead to an inconsistent solution in predicting quantum destructive interference effects that have profound relations to the wave mixing process. In this study, we describe an approximate analytical solution to a four-state double- $\Lambda$ scheme that is different from those studied previously [2-4]. Three features distinguish the present study from previous works: (1). A self-consistent fully time-dependent treatment leads to a three-photon destructive interference that extinguishes the laser excitation to the terminal state of a three-photon resonance, yet still provides nearly $25 \%$ frequency conversion efficiency. Such a three-photon destructive interference together with a high conversion efficiency from a quenched FWM state have not previously been reported in any double- $\Lambda$ scheme in the context of EIT, and cannot be obtained from a simple steadystate treatment. (2). We show that resonance excitation (which leads to perfect one-photon EIT) is not the desired condition for optimum production of the mixing wave. We point out that sizable detunings from these resonances are required in order to avoid the three-photon destructive interference that limits the conversion efficiency, and (3) We show that, contrary to common beliefs, high conversion efficiency is not determined by having the maximum atomic coherence between the states $\mid 0>$ and $\mid 1>$. Indeed, in this four-wave mixing scheme very little depletion of the ground state occurs and $\rho_{01}$ remains very small throughout the probe pulse. The correct criteria for achieving high conversion efficiency is a combination of the conventional phase-matching condition and a condition that ensures the constructive interference between two terms in the expression for the FWM field. In order to make both of the two terms of comparable size, a relatively large detuning is required to reduce near resonance absorption of one part of the four-wave mixing field.

\section{THEORETICAL MODEL}

Consider a four-level system that interacts with two transform-limited lasers $\left(E_{L 1}\left(\omega_{L 1}\right)\right.$ and $E_{L 2}\left(\omega_{L 2}\right)$, pulse length $\tau_{0}$, Fig.1). A short pulse probe laser (pulse length $\tau<<\tau_{0}$ ) is tuned near the $|0>\rightarrow| 2>$ resonance and fired at a 
predetermined delay time. We assume that during the pulse length of the probe laser the two long pulsed lasers at $\omega_{L 1}$ and $\omega_{L 2}$ are sufficiently powerful to strongly saturate $|2>\rightarrow| 1>$ and $|1>\rightarrow| 3>$ transitions, therefore, $\left|\Omega_{12} \tau\right|>>1$ and $\left|\Omega_{13} \tau\right|>>1$. As usual, $\Omega_{i j}=D_{i j} E_{L} /(2 \hbar)$ and $E_{L}$ are the one half Rabi frequency and the amplitude of the field for the respective transitions. Our objective is to seek a perturbative treatment for the response of the system to the short pulsed laser and to investigate the dynamics of the generated wave.

We start with three equations of motion for the amplitudes of atomic wave function in the non-depleted ground state approximation. We thus require that $\left|\Omega_{02}\right|<<\left|\Omega_{21}\right|$, so that most of the population remains in state $\mid 0>$. Taking $A_{0} \simeq 1$, we have

$$
\begin{aligned}
& \frac{\partial A_{1}}{\partial t}=i \Omega_{12} A_{2}+i \Omega_{13} A_{3}+i\left(\delta_{1}+i \frac{\gamma_{1}}{2}\right) A_{1}, \\
& \frac{\partial A_{2}}{\partial t}=i\left(\delta_{2}+i \gamma_{2} / 2\right) A_{2}+i \Omega_{20}+i \Omega_{21} A_{1}, \\
& \frac{\partial A_{3}}{\partial t}=i\left(\delta_{3}+i \gamma_{3} / 2\right) A_{3}+i \Omega_{31} A_{1}+i \Omega_{30} .
\end{aligned}
$$

In Eqs.(1), $\delta_{1}$ is the detuning from the two-photon resonance between $\mid 0>$ and $\mid 1>, \delta_{2}$ is the detuning of the short pulse probe laser from the $|0>\rightarrow| 2>$ resonance, $\delta_{3}$ is the detuning from the three-photon resonance involving the transition $|0>\rightarrow| 3>$. We will eventually assume that the lasers are tuned to the two-photon resonance $|0>\rightarrow| 1>$ and that the lifetime of this state is very long. Bear in mind that the generation of the four-wave mixing field will be shown to be very efficient, therefore, we must solve the above three equations of motion simultaneously with Maxwell's equations for both the probe and the generated fields. Taking unfocused beams and introducing $\kappa_{i j}=2 \pi \omega_{j i} N\left|D_{i j}\right|^{2} /(\hbar c)$ where $N$ is the concentration in $\mathrm{cm}^{-3}$, Maxwell's equations for these fields in slowly varying amplitude and phase approximation can be expressed as

$$
\begin{aligned}
& \frac{\partial \Omega_{20}}{\partial z}+\frac{1}{c} \frac{\partial \Omega_{20}}{\partial t}=i \kappa_{02} A_{2}, \\
& \frac{\partial \Omega_{30}}{\partial z}+\frac{1}{c} \frac{\partial \Omega_{30}}{\partial t}=i \kappa_{03} A_{3} .
\end{aligned}
$$

Notice that in the present model, there exist two very different time scales, i.e. $\tau<<\tau_{0}$, therefore, if the probe pulse occurs at the peak of the long pulse lasers, the amplitudes of the latter will remain nearly constant through the entire probe pulse. We therefore will be seeking a fully time dependent response of the system during the period when the probe pulse is present while treating both long pulse laser fields as time independent quantities. With this method in mind, Eqs.(1) and (2) can be solved analytically. Taking Fourier transform on the both sides of Eqs.(1) and (2), we obtain

$$
\begin{aligned}
\Omega_{21} \alpha_{1}+\left(\delta_{2}+\omega+i \gamma_{2} / 2\right) \alpha_{2} & =-W_{20}, \\
\left(\delta_{1}+\omega+i \gamma_{1} / 2\right) \alpha_{1}+\Omega_{12} \alpha_{2}+\Omega_{13} \alpha_{3} & =0, \\
\Omega_{31} \alpha_{1}+\left(\delta_{3}+\omega+i \gamma_{3} / 2\right) \alpha_{3} & =-W_{30}, \\
\frac{\partial W_{20}}{\partial z}-i \frac{\omega}{c} W_{20} & =i \kappa_{02} \alpha_{2}, \\
\frac{\partial W_{30}}{\partial z}-i \frac{\omega}{c} W_{30} & =i \kappa_{03} \alpha_{3} .
\end{aligned}
$$

where $\alpha_{1}, \alpha_{2}, \alpha_{3}, W_{20}$ and $W_{30}$ are the Fourier transforms of $A_{1}, A_{2}, A_{3}, \Omega_{20}$ and $\Omega_{30}$, respectively. Eqs. (3a-3c) can be solved in terms of $W_{20}$ and $W_{30}$ with the result

$$
\begin{aligned}
& \alpha_{1}=-\frac{D_{3} \Omega_{12}}{\Delta} W_{20}-\frac{D_{2} \Omega_{13}}{\Delta} W_{30}, \\
& \alpha_{2}=-\frac{\Omega_{21} \Omega_{13}}{\Delta} W_{30}+\frac{\left|\Omega_{13}\right|^{2}-D_{1} D_{3}}{\Delta} W_{20}, \\
& \alpha_{3}=-\frac{\Omega_{31} \Omega_{12}}{\Delta} W_{20}+\frac{\left|\Omega_{12}\right|^{2}-D_{1} D_{2}}{\Delta} W_{30},
\end{aligned}
$$

where,

$$
\begin{aligned}
D_{1} & =\delta_{1}+\omega+i \gamma_{1} / 2, \\
D_{2} & =\delta_{2}+\omega+i \gamma_{2} / 2, \\
D_{3} & =\delta_{3}+\omega+i \gamma_{3} / 2, \\
\Delta & =D_{1} D_{2} D_{3}-D_{3}\left|\Omega_{12}\right|^{2}-D_{2}\left|\Omega_{13}\right|^{2} .
\end{aligned}
$$


When these equations are used in Eqs (3d-3e), we obtain

$$
\begin{aligned}
& \frac{\partial W_{20}}{\partial z}-i \frac{\omega}{c} W_{20}=i \kappa_{02} \frac{\left(\left|\Omega_{13}\right|^{2}-D_{1} D_{3}\right)}{\Delta} W_{20}-i \kappa_{02} \frac{\Omega_{21} \Omega_{31}}{\Delta} W_{30}, \\
& \frac{\partial W_{30}}{\partial z}-i \frac{\omega}{c} W_{30}=i \kappa_{03} \frac{\left(\left|\Omega_{12}\right|^{2}-D_{1} D_{2}\right)}{\Delta} W_{30}-i \kappa_{03} \frac{\Omega_{31} \Omega_{12}}{\Delta} W_{20} .
\end{aligned}
$$

For given $W_{20}(0, \omega)$ and with $W_{30}(0, \omega)=0$, Eqs $(5)$ can be solved analytically, yielding

$$
\begin{aligned}
& W_{30}(z, \omega)=i \frac{W_{20}(0, \omega) S_{3}}{\Lambda} e^{i D z} \sin (\Lambda z) \\
& W_{20}(z, \omega)=\frac{W_{20}(0, \omega)}{\Lambda} e^{i D z}\left(i \frac{K_{2}-K_{3}}{2} \sin (\Lambda z)+\Lambda \cos (\Lambda z)\right),
\end{aligned}
$$

where we have defined the new parameters

$$
\begin{aligned}
\Lambda & =\sqrt{\left(\frac{K_{2}-K_{3}}{2}\right)^{2}+S_{2} S_{3}}, \\
D & =\frac{K_{2}+K_{3}}{2}, \\
K_{2} & =\frac{\omega}{c}+\kappa_{02} \frac{\left|\Omega_{13}\right|^{2}-D_{1} D_{3}}{\Delta}, K_{3}=\frac{\omega}{c}+\kappa_{03} \frac{\left|\Omega_{12}\right|^{2}-D_{1} D_{2}}{\Delta}, \\
S_{2} & =-\kappa_{02} \frac{\Omega_{21} \Omega_{13}}{\Delta}, S_{3}=-\kappa_{03} \frac{\Omega_{31} \Omega_{12}}{\Delta} .
\end{aligned}
$$

If we use, for the probe laser at the entrance to the medium,

$$
\Omega_{02}(0, t)=\Omega_{02}(0,0) e^{-(t / \tau)^{2}},
$$

we find

$$
W_{02}(0, \eta) / \Omega_{02}(0,0)=\frac{\tau}{\sqrt{2}} e^{-\eta^{2} / 4},
$$

where we have introduced the dimensionless variable $\eta=\omega \tau$. We then get

$$
\begin{aligned}
& \Omega_{30}\left(z, \frac{t}{\tau}\right)=\frac{i \Omega_{20}(0,0)}{\sqrt{4 \pi}} \int_{-\infty}^{\infty} d \eta e^{-\frac{\eta^{2}}{4}} e^{i D(\eta) z} e^{-i \eta \frac{t}{\tau}} S_{3}(\eta) \frac{\sin (\Lambda z)}{\Lambda} \\
& \Omega_{20}\left(z, \frac{t}{\tau}\right)=\frac{\Omega_{20}(0,0)}{\sqrt{4 \pi}} \int_{-\infty}^{\infty} d \eta e^{-\frac{\eta^{2}}{4}} e^{i D(\eta) z} e^{-i \eta \frac{t}{\tau}}\left[i \frac{K_{2}-K_{3}}{2 \Lambda} \sin (\Lambda z)+\cos (\Lambda z)\right] .
\end{aligned}
$$

In the following section, we will discuss the physical implications of Eq.(8) by examining some limiting cases where Eq.(8) can be evaluated analytically. In Section IV, we will evaluates Eqs. (8) numerically and compare the results with the analytical approximations.

\section{Discussions}

In this section we focus on the physical interpretation of Eqs.(8). We will consider some limiting cases where the inverse transform of the generated field can be carried out analytically. These limiting cases provide a great deal of insight into the wave propagation effects.

Consider the limiting case where $\left|\Omega_{12}\right|,\left|\Omega_{13}\right|>>\left|\delta_{1}\right|,\left|\delta_{2}\right|,\left|\delta_{3}\right|, \gamma_{1}, \gamma_{2}, \gamma_{3}$. In fact, in what follows we shall always use $\delta_{1}=0$ and assume that state $\mid 1>$ is a second hyperfine level of the ground state, so that in an ultra-cold vapor $\gamma_{1} \tau<<1$. In this case the width of state $\mid 1>$ is determined by the very slow rate of collisions between the atoms of cold low density vapor. Under these conditions, an accurate approximation to the parameters in Eqs. (7) can be obtained by assuming $\left|\Delta_{1}\right|<<\left|\Delta_{2}\right|, \quad\left|\Delta_{3}\right|$, and $\left|\Omega_{12}\right|,\left|\Omega_{13}\right|>>\left|\Delta_{2}\right|,\left|\Delta_{3}\right|$. Within this limit we can expand $\Lambda$ by making use of the assumption that either $\left|\Omega_{13}\right|^{2}$, or $\left|\Omega_{12}\right|^{2}$ is much larger than either $\left|D_{1} D_{2}\right|$ or $\left|D_{1} D_{3}\right|$, therefore,

$$
\frac{K_{2}+K_{3}}{2}-\sqrt{\left(\frac{K_{2}-K_{3}}{2}\right)^{2}+S_{2} S_{3}} \simeq-D_{1} \frac{\kappa_{12} \kappa_{32}}{\kappa_{12}\left|\Omega_{13}\right|^{2}+\kappa_{32}\left|\Omega_{12}\right|^{2}} .
$$


If we replace sine and cosine in the inverse transform by complex exponentials, this approximation allows us to evaluate part of the integrals analytically for arbitrary pulse shape. When the detunings $\delta_{2}$ and $\delta_{3}$ are very small compared with the half-Rabi frequencies, the other integrals are damped out in a small distance due to absorption.

We first analyze the case where $\delta_{1}=\delta_{2}=\delta_{3}=0$, i.e. all lasers are tuned exactly on the unperturbed resonances. This is the limit where one-photon EIT is achieved. In this limit we find that after a large propagation distance, and at a point where the FWM has built up sufficiently

$$
\begin{aligned}
& \Omega_{30}(z, t)=\frac{\kappa_{03} \Omega_{31} \Omega_{12}}{\kappa_{02}\left|\Omega_{13}\right|^{2}+\kappa_{03}\left|\Omega_{12}\right|^{2}} \Omega_{20}\left(0, t-\frac{z}{V_{g 1}}\right), \\
& \Omega_{20}(z, t)=\frac{\kappa_{03}\left|\Omega_{12}\right|^{2}}{\kappa_{02}\left|\Omega_{13}\right|^{2}+\kappa_{03}\left|\Omega_{12}\right|^{2}} \Omega_{20}\left(0, t-\frac{z}{V_{g 1}}\right),
\end{aligned}
$$

where

$$
\frac{1}{V_{g 1}}=\frac{1}{c}+\frac{\kappa_{12} \kappa_{32}}{\kappa_{12}\left|\Omega_{13}\right|^{2}+\kappa_{32}\left|\Omega_{12}\right|^{2}} .
$$

This result indicates that in this limit $\Omega_{20}(z, t)=\left(\Omega_{21} / \Omega_{31}\right) \Omega_{30}(z, t)$.[5] Indeed, Eq. (4c) shows that in this limit $\alpha_{3} \simeq 0$ for all $\omega$, providing $z$ is large enough to make $\left.\mid \exp \left(i\left[\kappa_{02}\left|\Omega_{13}\right|^{2}+\kappa_{03}\left|\Omega_{12}\right|^{2}\right] / \Delta\right]\right) \mid<<1$. It is required that $\left|\Omega_{12}\right|^{2}>>\left|D_{1} D_{2}\right|$ in order for $W_{02} / W_{03}=\Omega_{12} / \Omega_{13}$ to imply that $\alpha_{3}=0$. In other words, a destructive interference has occured between the excitation pathways for the state. [6] We emphasize that this suppression of the excitation of the state $\mid 3>$ cannot be obtained from the usual steady-state treatment of the atomic equations of motion. Indeed, the condition derived in the steady-state frame work for a three-photon destructive interference leads to an inconsistent prediction of the effect. We have evaluated the special case where $\kappa_{12} \tau=\kappa_{23} \tau=200 \mathrm{~cm}^{-1},\left|\Omega_{12} \tau\right|=5$, $\left|\Omega_{13} \tau\right|=20, \gamma_{1} \tau=0.02, \gamma_{2} \tau=\gamma_{3} \tau=2$ using both Eq.(10) and numerical integration of Eq.(8). As will be seen later the approximate analytical solution is in excellent agreement with the numerical evaluation and the ratio of $\Omega_{20}(z, t) / \Omega_{30}(z, t)$ at $z=10 \mathrm{~cm}$ is equal to a constant (at all $\mathrm{t}$ where the two quantities are greater than $10^{-4}$ of their peak values) to an accuracy of seven significant figures. To this accuracy, the constant ratio is equal to $\Omega_{12} / \Omega_{13}$. Also, the peak in the two half-Rabi frequencies occurs at the point predicted by the group velocity $V_{g 1}$. Note that if one allows $\left|\Omega_{13}\right|=0$ then $V_{g 1}$ reduces to the expression appropriate to the extremely slow wave propagation experiment [8], as should be the case.

It should be pointed out that the destructive interference predicted above is equivalent to EIT for both the four-wave mixing photons and the probe laser photons. To see this clearly, consider the condition for Eq. (1b) to predict $A_{2}=0$ at a point in space. This would be the case if $A_{1}=-\Omega_{20} / \Omega_{21}$ at all times. With this amplitude for $A_{1}$ the coupling terms destructively interfere. This is what happens when EIT occurs for the probe laser, for in this case there is no polarization to lowest order at $\omega_{p}$. Correspondingly, in order to have EIT at the four-wave mixing frequency we must have $A_{3}=0$. Looking at Eq. (1c), we see that this requires $A_{1}=-\Omega_{30} / \Omega_{31}$. In order for EIT to occur at both frequencies, the two values of $A_{1}$ must be the same. This yields $\Omega_{20} / \Omega_{21}=\Omega_{30} / \Omega_{31}$. The same relation holds in Eqs. (10).

We now consider the situation where $\left|\Omega_{12}\right| \tau=\left|\Omega_{13}\right| \tau>100,\left|\delta_{1}\right|=0$ and $\gamma_{1} \tau<<1$. We also assume that $\left|\delta_{3}\right| \tau>>1$, but $\left|\delta_{2} / \Omega_{12}\right|^{2},\left|\delta_{3} / \Omega_{12}\right|^{2},\left|\delta_{2} / \Omega_{13}\right|^{2},\left|\delta_{3} / \Omega_{13}\right|^{2} \leq 1$. These conditions indicate that both the Autler-Townes splittings and the detunings are large enough so that very little absorption occurs. In this limit three-photon destructive interference no longer occurs, and we find

$$
\begin{aligned}
\Omega_{30}(z, t)= & \frac{\kappa_{03} \Omega_{31} \Omega_{12}}{\kappa_{02}\left|\Omega_{13}\right|^{2}+\kappa_{03}\left|\Omega_{12}\right|^{2}}\left(\Omega_{20}\left(0, t-\frac{z}{V_{g 1}}\right)-\Omega_{20}\left(0, t-\frac{z}{V_{g}}\right) e^{i P z}\right), \\
\Omega_{20}(z, t)= & \frac{\kappa_{03}\left|\Omega_{12}\right|^{2}}{\kappa_{02}\left|\Omega_{13}\right|^{2}+\kappa_{03}\left|\Omega_{12}\right|^{2}} \\
& \quad \times\left(\Omega_{20}\left(0, t-\frac{z}{V_{g 1}}\right)+\frac{\kappa_{02}\left|\Omega_{13}\right|^{2}}{\kappa_{03}\left|\Omega_{12}\right|^{2}} \Omega_{20}\left(0, t-\frac{z}{V_{g}}\right) e^{i P z}\right)
\end{aligned}
$$

where we have introduced notations

$$
\begin{aligned}
P & =\frac{\kappa_{02}\left|\Omega_{13}\right|^{2}+\kappa_{03}\left|\Omega_{12}\right|^{2}}{\left|\Omega_{12}\right|^{2} \delta_{3}+\left|\Omega_{13}\right|^{2} \delta_{2}}, \\
\frac{1}{V_{g}} & =\frac{1}{c}+\frac{\left(1+\left|\Omega_{13} / \Omega_{12}\right|^{2}\right)\left(\kappa_{02}\left|\Omega_{13} / \Omega_{12}\right|^{2}+\kappa_{03}\right)}{\left(\delta_{3}+\left|\Omega_{13} / \Omega_{12}\right|^{2} \delta_{2}\right)^{2}} .
\end{aligned}
$$


In the expression of $V_{g}$ we have neglected a term that is of the same order of magnitude as $1 / V_{g 1}-1 / c$, since such a term is much smaller than $1 / V_{g}-1 / c$ in the above equation when the half-Rabi frequencies are much larger than the detunings. Eq.(11) indicates that there are two contributions to the growth of the FWM field $\Omega_{30}$. The first contribution is due to the probe field that travels at the group velocity $V_{g 1}$, whereas the second term consists of a probe field that travels at a second group velocity, $V_{g}$. If these two parts do not separate appreciably before a distance $z$ such that $|P z|=\pi$ is reached, the two parts will interfere constructively. In the case where $\left|\Omega_{13} / \Omega_{12}\right| \simeq 1$, this requires that $c\left(\kappa_{02}+\kappa_{03}\right)<<\left(\delta_{2}+\delta_{3}\right)^{2}$ and $c / V_{g 1} \simeq 1$. The latter condition puts restrictions on $k a_{12} c \tau^{2}$ and $\kappa_{32} c \tau^{2}$, as compared with $\left|\Omega_{12} \tau\right|$ and $\left|\Omega_{13}\right| \tau$.

The photon flux conversion efficiency under this condition can be found as

$$
\frac{F_{m}}{F_{p}}=\frac{\kappa_{03}\left|\Omega_{12}\right|^{2} \kappa_{02}\left|\Omega_{13}\right|^{2}}{\left(\kappa_{03}\left|\Omega_{12}\right|^{2}+\kappa_{02}\left|\Omega_{13}\right|^{2}\right)^{2}}\left|e^{-(t-z / c)^{2} / \tau^{2}}-e^{i P z-Q z-\left(t-z / V_{g}\right)^{2} / \tau^{2}}\right|^{2},
$$

where

$$
Q=P \frac{\left|\Omega_{12}\right|^{2} \gamma_{3} / 2+\left|\Omega_{13}\right|^{2} \gamma_{2} / 2}{\left|\Omega_{12}\right|^{2} \delta_{3}+\left|\Omega_{13}\right|^{2} \delta_{2}}
$$

In Eq.(12), $F_{m}$ and $F_{p}$ are the photon fluxes for the FWM and probe fields, respectively, and in deriving this expression we have, for the mathematical simplicity, assumed a Gaussian pulse shape for the probe field so that $W_{20}(0, \omega)=\frac{\Omega_{20}(0,0) \tau}{\sqrt{2}} e^{-\omega^{2} \tau^{2}}$. ¿From Eq.(12), we notice that if we choose $|P z|=\pi$ and $\kappa_{02}\left|\Omega_{13}\right|^{2}=\kappa_{03}\left|\Omega_{12}\right|^{2}$, then we obtain $F_{m} \simeq F_{p}$. This implies that the conversion efficiency is close to $100 \%$ whenever the difference between $V_{g 1}$ and $V_{g}$ is small and the absorbtion factor $|Q z|<<1$. Extensive numerical calculations have verified that conversion efficiencies of nearly $100 \%$ are indeed produced when the conditions stated before are well satisfied.

In the case where the three-photon destructive interference does occur, i.e. in the case when perfect one-photon EIT is established with $\delta_{1} \tau=\delta_{2} \tau=\delta_{3} \tau=0$, the second term in Eq.(12) disappears and the integral can be evaluated analytically. The result is a nearly $25 \%$ conversion efficiency in spite of the fact that the FWM field is strongly suppressed and there will be no further production of the field in the rest of medium. This indicates that for the scheme studied here, achieving one-photon EIT is detrimental to the high flux conversion efficiency. Therefore, one prefers to detune the two long pulsed lasers from the perspective resonances in order to avoid the destructive interference. Another important conclusion that can be immediately deduced from Eq.(9) is that it shows that maximum atomic coherence is not the optimum choice for maximum conversion efficiency as previously suggested [2]. Indeed, there are two conditions that must be met in order to achieve maximum conversion efficiency. The first one is the combination of $\kappa_{02}, \kappa_{03},\left|\Omega_{12}\right|$, and $\left|\Omega_{13}\right|$ that maximize the amplitude of the expression in Eqs.(10-12). It has been shown [7] that this is precisely the conventional phase-matching condition for efficient wave mixing process. The second condition that must be met is that the propagation distance must be chosen properly in order to make the two terms in Eq.(12) interfere constructively. It is the combination of these two conditions that enables a highly efficient wave mixing and flux conversion process.

\section{NUMERICAL SIMULATIONS}

In this section, we investigate the model system numerically. Our main focus is to study the validity of Eq.(12) under specified conditions. This expression gives a complete description, under the conditions prescribed, on the flux conversion efficient with the field propagation effect included. It contains all the functional dependence of the FWM field on time, propagation distance, Rabi frequencies and detunings. We will first compare Eq.(12) with direct numerical evaluation of the inverse transform in order to show the validity of our approximation that leads to analytical results. After having established the validity of the analytical result, we then investigate the functional dependence of the conversion efficiency on parameters such as detunings and pumping Rabi frequencies, which may be useful in experimental verification of the theory.

As mentioned before, the probe field propagation, generation of the FWM field, and the high-efficiency photon flux conversion described in the previous sections have all been verified through extensive numerical calculations. In Figure 2a, we first show, for a typical set of parameters, the conversion efficiency predicted by the approximation (Eq.(12)) and by direct numerical integration of the inverse transform as a function of $(t-z / c) / \tau$. The parameters are so chosen that the three-photon destructive interference is defeated, therefore, both terms in Eq.(12) contribute to the overall conversion efficiency. Furthermore, we have chosen the parameters that maximizes the amplitude of 
the expression given in Eq.(11). The graph shows an excellent agreement between the approximate solution and the numerical evaluation of the inverse transform, and both methods predict nearly $100 \%$ conversion efficiency. In Figure $2 \mathrm{~b}$, a different set of propagation parameters are chosen under the condition where the three-photon destructive interference is not present. Again, the plot shows an excellent agreement between the two methods. These results indicate that the analytical result Eq.(12) can, under the conditions given, correctly predict the propagation effect of the generated field. Therefore, we will use Eq.(12) to explore the functional dependence of the conversion efficient to various detunings and power densities. As has mentioned before, EIT process is established when both long pulse laser are tuned on resonance, i.e. $\delta_{2} \tau=\delta_{3} \tau=0$. The resulting conversion efficiency can be analytically obtained. In Figure $3 \mathrm{a}$, we show the dependence of the efficiency as function of $\delta_{2} \tau$. As the detuning of the second laser from the resonance is increased, the efficiency increases, as expected. The similar effect due to the detuning $\delta_{3} \tau$ is also shown in Figure 3b. These figures indicate that for optimum operation, one should choose non-vanishing detunings which also satisfy the conditions forth set for the Rabi frequencies. Finally, we investigate the dependence of the conversion efficiency on laser powers. First, it is obvious that Eq.(9) is independent of the power of the probe laser. In Figure 4 , we plot the efficiency as function of $\left|\Omega_{13} / \Omega_{12}\right|^{2}$. As expected, the maximum conversion efficiency is achieved only at $\left|\Omega_{13} / \Omega_{12}\right|^{2}=0.25$, a necessary condition, for a given $\kappa_{02} / \kappa_{03}=4$, in order to achieve phase matching for the generated weave.

\section{Conclusion}

In conclusion, we have presented an approximate analytical solution to a four-level double- $\Lambda$ scheme where laser induced transparency is expected. We predict that when the detunings from the three-photon resonance is small, and therefore the usual one-photon EIT process is established, the FWM field propagates without suffering any pulse distortion. Under these conditions, We also predict that at a sufficient propagation depth a complete threephoton destructive interference involving the FWM field and the three laser fields will reduce the polarization at the four-wave mixing frequency to zero. We have shown that even with such a robust three-photon destructive interference that strongly suppresses further generation of the FWM field beyond an onset propagation distance, the photon flux conversion efficiency can still be as high as $25 \%$. Our result, however, indicates that one should detune both pump lasers from the perspective resonances, thereby avoid the usual one-photon EIT process. The use of a significant detuning will defeat a possible three-photon destructive interference (that limits the further production of the internally generated wave and therefore limits conversion efficiency to $25 \%$ ) therefore, allowing photon flux conversion efficiencies close to $100 \%$ with even a very weak probe field. In addition, our calculation shows that, contrary to common belief, a maximum atomic coherence between the two lower states is not the optimum condition for achieving maximum conversion efficiency. Indeed, with the current scheme $\left|\rho_{01}\right|<<1$. For high conversion efficiency, proper atomic parameters should be chosen according to Eqs.(11) and (12).

\section{List of References}

1. S.E. Harris, Phys. Today 50, 36 (1997).

2. A.J. Merriam et al, Phys. Rev. Lett. 84, 5308 (2000), A.J. Merriam et al, IEEE J. SEL. TOP. QUANT. 5, 1512 (1999), A.J. Merriam et al, Opt.Lett 24, 625 (1999), M. Jain et al, Phys. Rev. Lett. 77, 4326 (1996).

3. M.D. Lukin et al, Phys. Rev. A 60, 3225 (1999). For more works related to double- $\Lambda$ scheme, see M.D. Lukin, P.R. Hemmer, and M.O. Scully, Adv. in Atm. Mol. and Opt. Phys., Vol 42, 347 (2000) and reference therein.

4. Early studies on double- $\Lambda$ system coupled with laser excitations can be found in S.J. Buckle et al., Opta. Acta 33, 1129 (1986). This study assumed that four externally generated laser fields, with their phase properties being precisely controlled, couples all four levels, therefore, no generated field is involved. It is not surprising that with precise control of the phase of all laser field, a two-photon cancellation is predicted by this study. Since this steady-treatment does not include the light propagation, therefore could not predict any propagation based interference effect as demonstrated in the present study. The same system was later re-examined by E.A. Korsunsky et al., Phys. Rev. A60, 4996 (1999) with the extension to include the steady-state Maxwell equation for laser fields. It also includes discussions on coherent population transfer and induced transparency. However, due to its CW nature, the study could not provide any dynamics of the system. In addition, the treatment is valid only when all four laser frequencies are close to each other. Both studies could not predict, due to the $\mathrm{CW}$ nature of the treatments, a possible three-photon destructive interference that must involve a phase matched internally generated field.

5. This relation is similar to that obtained in the two references listed in Ref.[4]. However, it is important to understand the difference between our results and that given in Ref.[4]. For a four-level system coupled with 
four lasers with precise phase control, as described in Ref[4], one can make such destructive interference work for a wide range of detunings. In the case where an internally generated wave contribute to the interference, however, the robust cancellation effect can happen only when the phase matching condition for the efficient generation of the mixing wave is fulfilled. Further, it is fundamentally different that in the case where the generated field is excluded, as in Ref. [4], there is no appreciable effect due to wave propagation, therefore can only be achieved at very low concentration such as atomic beam. In the case where the mixing wave participates in the process, the destructive interference is readily observable even at elevated concentrations.

6. M.G. Payne, L. Deng, and W.R. Garrett, Phys. Rev. A 58, 1361 (1998); L. Deng, M.G. Payne, and W.R. Garrett, Phys. Rev. A 52, 489 (1995); R.C. Hart et al., Phys. Rev. A 46, 4213 (1992); M.G. Payne et al., Phys. Rev. A 44, 7684 (1991); J.C. Miller et al., Phys. Rev. Lett. 45, 114 (1980).

7. L. Deng, M.G. Payne, and W.R. Garrett, Phys. Rev. A 63, 3811 (2001); L. Deng, M.G. Payne, and W.R. Garrett, Phys. Rev. A 64, 31802R (2001).

8. L.V. Hau et al., Nature (London) 397, 594 (1999).

Figure Captions

Figure 1: Energy level diagram showing a four-level double- $\Lambda$ scheme with relevant laser couplings. The theory and predictions presented in the text are expected to hold with minor modifications for other orderings of the energies of the excited states.

Figure 2a: A plot of conversion efficiency comparing approximate solution and direct integration of the inverse transform as a function of $(t-z / c) / \tau$. Parameters used: $\left|\Omega_{12}\right| \tau=200,\left|\Omega_{13}\right| \tau=100, \kappa_{03} c \tau^{2}=10, \kappa_{02} c \tau^{2}=40$, $\gamma_{1} \tau=\gamma_{2} \tau=\gamma_{3} \tau=0.1, \delta_{1} \tau=0, \delta_{2} \tau=20, \delta_{3} \tau=20$, and $z=3.93 \mathrm{~cm}$ for constructive interference. The solid line is the approximate solution whereas the solid circles are full numerical solutions. Nearly $100 \%$ conversion efficiency is predicted by both methods.

Figure 2b: Same plot as in Figure 2a except $\delta_{1} \tau=0, \delta_{2} \tau=10, \delta_{3} \tau=10$, and $z=1.96 \mathrm{~cm}$ for constructive interference. The solid line is the approximate solution whereas the solid circles are full numerical solutions.

Figure 3a: Flux conversion efficiency vs. a dimensionless quantity $\eta=(t-z / c) / \tau$ for a set of different detuning $\delta_{2} \tau$. Open circle: $\delta_{2} \tau=10$, solid triangle: $\delta_{2} \tau=20$, open diamond: $\delta_{2} \tau=40$, solid circle: $\delta_{2} \tau=60$. All other parameters are the same as in Figure 2a.

Figure 3b: Flux conversion efficiency vs. a dimensionless quantity $\eta=(t-z / c) / \tau$ for a set of different detuning $\delta_{3} \tau$. Open circle: $\delta_{3} \tau=10$, solid triangle: $\delta_{3} \tau=20$, open diamond: $\delta_{3} \tau=40$, solid circle: $\delta_{3} \tau=60$. All other parameters are the same as in Figure 2a.

Figure 4: Flux conversion efficiency vs. a dimensionless quantity $\eta=(t-z / c) / \tau$ for a set of different ratio of Rabi frequencies $\left|\Omega_{13} / \Omega_{12}\right|^{2}$. Cross: $\left|\Omega_{13} / \Omega_{12}\right|^{2}=1$, open circle: $\left|\Omega_{13} / \Omega_{12}\right|^{2}=0.5$, solid circle: $\left|\Omega_{13} / \Omega_{12}\right|^{2}=0.25$, and open triangle: $\left|\Omega_{13} / \Omega_{12}\right|^{2}=0.1$. All other parameters are the same as in Figure 2a. 
This figure "f1.jpg" is available in "jpg" format from: http://arxiv.org/ps/quant-ph/0203076v1 
This figure "f2.jpg" is available in "jpg" format from: http://arxiv.org/ps/quant-ph/0203076v1 
This figure "f3.jpg" is available in "jpg" format from: http://arxiv.org/ps/quant-ph/0203076v1 
This figure "f4.jpg" is available in "jpg" format from: http://arxiv.org/ps/quant-ph/0203076v1 